\title{
A new spinwave expansion for the ordered Kondo lattice
}

\author{
Nic Shannon \\ Max-Planck-Institut für Physik komplexer Systeme, Nöthnitzer Str. 38, 01187 Dresden, Germany
}

\begin{abstract}
We present a concise introduction to a new spinwave expansion scheme for magnetically ordered Kondo lattice models. This is motivated by consideration of the ferromagnetically ordered phase of the "double exchange" system $\mathrm{La}_{1-x} \mathrm{Ca}_{x} \mathrm{MnO}_{3}$. A brief overview is given of the consequences of quantum and thermal fluctuation effects for the magnetic properties of the double exchange ferromagnet.
\end{abstract}

\section{INTRODUCTION}

The defining feature of transition metals is mixed valence - their ability to donate a different numbers of electrons to chemical bonds according to the detailed charge balance of their environment. In addition, magnetic transition metals like Manganese have a strong Hund's first rule coupling. This forces all electrons in the unfilled shell of the ion to align their spin, giving rise to a net local magnetic moment.

Taken together, these two facts have important consequences for magnetism in doped transition metal oxides, where the transition metal ions can frequently be found in states with different valence and therefore different total spin. Where the electrons are also able to move from site to site, local moment behaviour normally associated with magnetic insulators can coexist with metallic conduction. In this article we will give a brief pedagogical overview of a systematic method of describing the magnetic and charge excitations of such as a system. The approach developed has the conceptual and technical advantage that it does not introduce any artificial distinction between the magnetism of itinerant and localized electrons

\section{FROM MATERIAL TO MODEL}

A good example of a mixed valent system with strong Hund's rule coupling is provided by the family of "colossal magnetoresistance" manganites $\mathrm{La}_{1-x} \mathrm{Ca}_{x} \mathrm{MnO}_{3}$. This compound has a perovskite structure, like the High Temperature superconductors, and the magnetic manganese atoms occupy sites on a cubic lattice - see Fig. 1. We can rewrite the chemical formula of this manganite as $\left(\mathrm{La}^{3+} \mathrm{Mn}^{3+}\right)_{1-x}\left(\mathrm{Ca}^{2+} \mathrm{Mn}^{4+}\right)_{x}\left(\mathrm{O}^{2-}\right)_{3}$. Strong Hund's rule coupling then means that for each unit cell the system contains $1-x$ spin $S=2$ local moments (the $\mathrm{Mn}^{3+}$ ions), and $x$ spin $S=3 / 2$ local moments ( $\mathrm{Mn}^{4+}$ ions). States with an integer number of electrons per Mn site cannot conduct, and the parent 


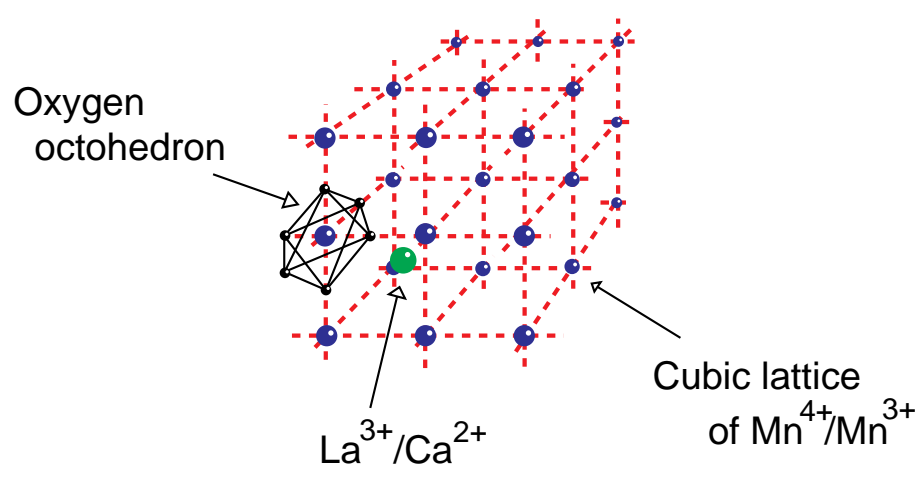

FIGURE 1. Cubic lattice of $\mathrm{Mn}$ ions in $\mathrm{La}_{1-x} \mathrm{Ca}_{x} \mathrm{MnO}_{3}$.

compound $\mathrm{LaMnO}_{3}$ is a spin $S=2$ antiferromagnetic (AF) insulator.

On doping with $\mathrm{Ca}$, "holes" are introduced onto the $\mathrm{Mn}$ sites, so that the system now has a mixture of $S=2 \mathrm{Mn}^{3+}$ and $S=3 / 2 \mathrm{Mn}^{4+}$ ions. The crystal field at the Mn sites splits $\mathrm{Mn} d$-electron levels into a $t_{2 g}$ and an $e_{g}$ multiplet, as shown in Fig. 2. Where the $e_{g}$ electrons delocalize, this leads to a natural description of the material in terms of localized and itinerant electrons.

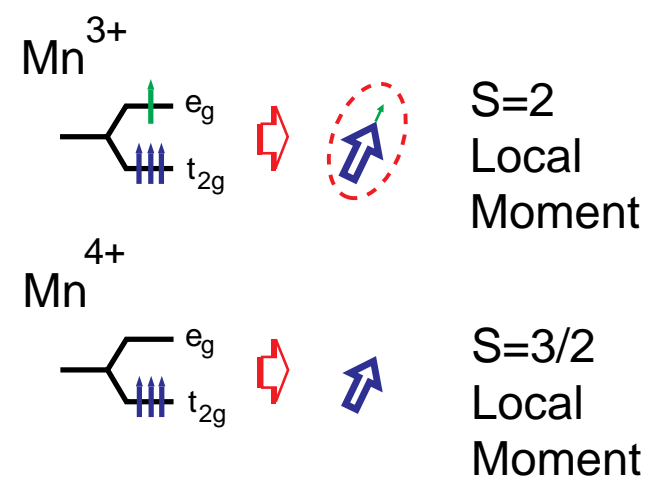

FIGURE 2. Crystal field splitting and $\mathrm{Mn}$ spin states in $\mathrm{La}_{1-x} \mathrm{Ca}_{x} \mathrm{MnO}_{3}$.

The simplest model Hamiltonian we can use to describe such a system is the Kondo lattice model

$$
\mathcal{H}=-t \sum_{\langle i j\rangle \alpha} c_{i \alpha}^{\dagger} c_{j \alpha}-J \sum_{i} \vec{s}_{i} \cdot \vec{s}_{i} \quad \vec{s}_{i}=\frac{1}{2} \sum_{\alpha \beta} c_{i \alpha}^{\dagger} \overrightarrow{\sigma_{\alpha \beta}} c_{i \beta}
$$

This model has already been much discussed during the course of this school, usually for small $S=1 / 2$ and in the limit $|J| \ll t$ where $J<0$ is the AF Kondo coupling. Here we must consider the opposite limit of $t \ll|J|$ for ferromagnetic (FM) $J>0$. We will consider only states in which the system is magnetically ordered, in which case it is reasonable to assume that the size of the local moment $S \gg 1$. For simplicity, we neglect the degeneracy of the itinerant electron $e_{g}$ orbital. 


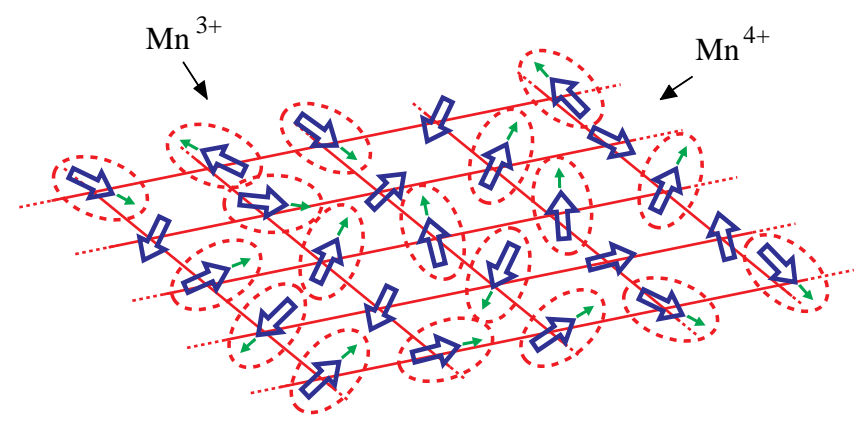

FIGURE 3. Paramagnetic state in the absence of inter site hopping $(t=0)$.

Since $t / J$ is a small parameter it makes sense to diagonalize the Hund's rule coupling term $\propto J$ first, and then to reintroduce the kinetic energy $\propto t$. In the absence of any coupling between sites $(t=0)$, for any density of holes, the system must be an insulator with paramagnetic Curie law susceptibility - see Fig 3 . For finite $t>0$ the ground state, at least at large $S$, will be a metallic FM, since the band kinetic energy of the itinerant electrons is greatest when all the spins are aligned - see Fig 4 . This effect is was named "double exchange" by Zener [1], because in $\mathrm{La}_{1-x} \mathrm{Ca}_{x} \mathrm{MnO}_{3}$, itinerant electrons move between from one Mn site to another through connecting $0_{2 p}$ orbitals. Where such a FM groundstate is realized, it is therefore referred to as a double exchange ferromagnet (DEFM).

In what follows we will develop a controlled expansion scheme for calculating the properties of the localized/itinerant electron system described by Eqn. [1. Here we will only discuss the DEFM, but the methods developed are much more general and may be applied to any magnetically ordered Kondo lattice model.

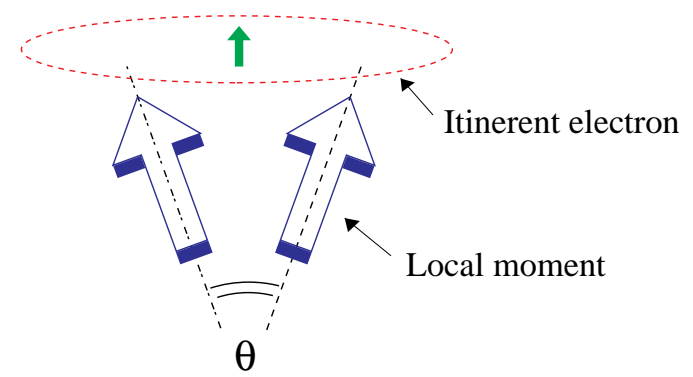

FIGURE 4. A single double exchange bond in the limit $J \rightarrow \infty, S \rightarrow \infty$. 

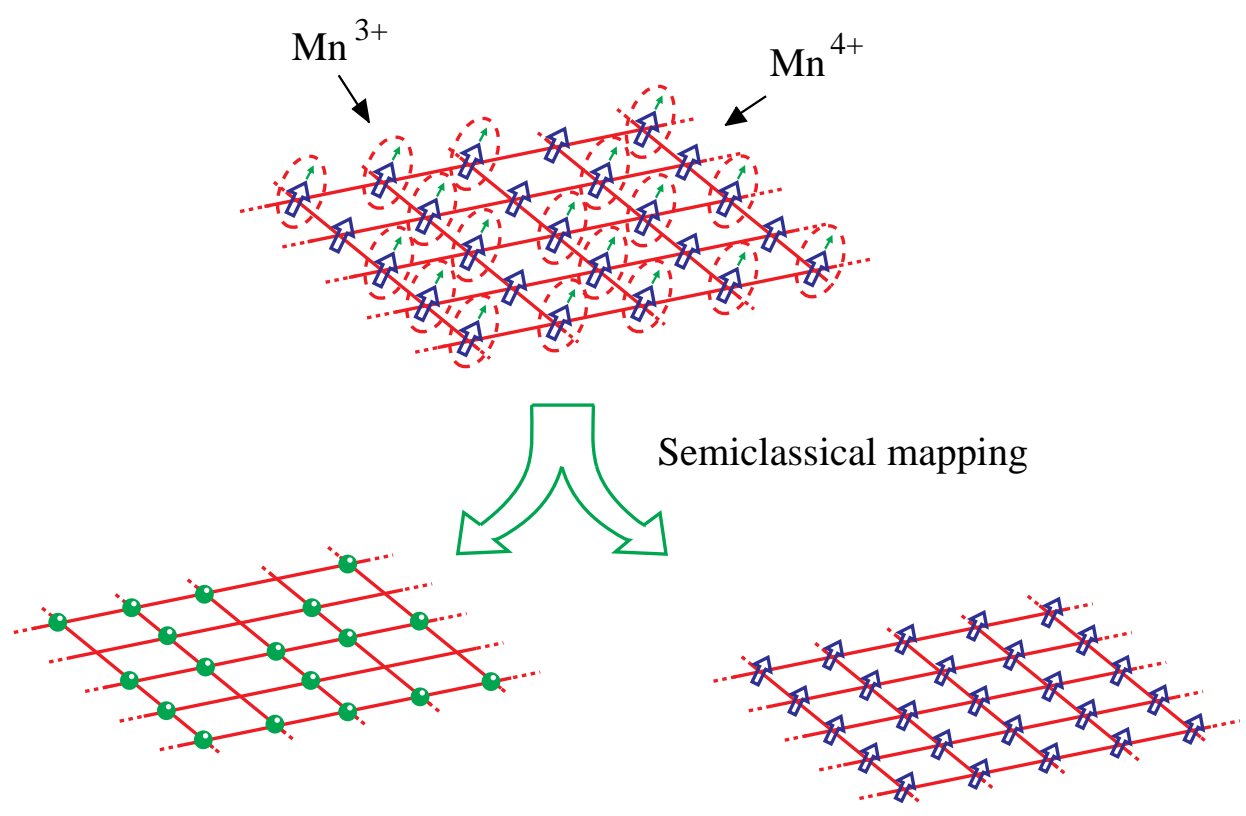

Charge degrees of freedom

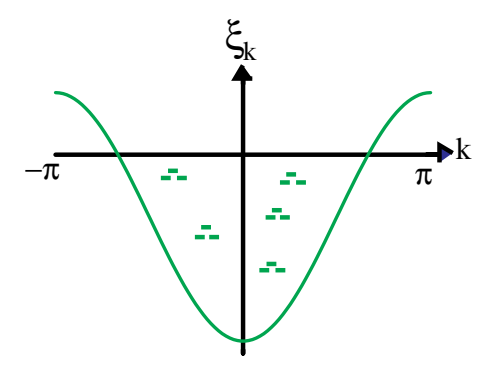

Spin degrees of freedom

Tight-binding band of spinless electrons

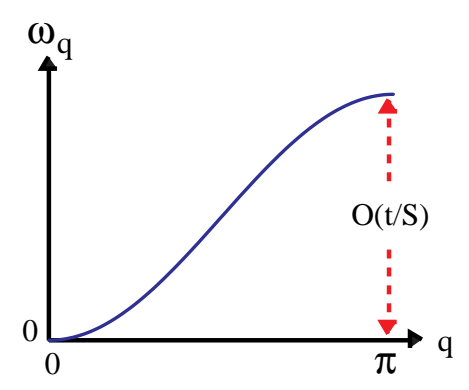

Band of ferromagnetic spinwave excitations

FIGURE 5. DEFM for $t>0$ and semiclassical mapping onto independent spin and charge excitations in limit $J \rightarrow \infty, S \rightarrow \infty$.

\section{SEMI-CLASSICAL TREATMENT}

The physics of the DEFM can be understood easily at a semi-classical level, by analogy with the problem of two local moments sharing a single electron. This was first solved by Anderson and Hasegawa [2], who considered the Hamiltonian Eqn. 1] restricted to two sites. In the limit $S \rightarrow \infty$ we can define an angle $\theta$ between the two local moments 
(Fig. 5). The energy of the ground state for a given $\theta$ is given by

$$
E_{0}=-\frac{J S}{2}-t \cos \left(\frac{\theta}{2}\right)
$$

where an $S U(2)$ rotation of the local spin quantization axis, together with the limit $J \rightarrow \infty$, has been used to project out those electron states which are not aligned with the local moment on each site. There are two contributions to this an energy, a term proportional to $J$ which is the Hund's first rule binding energy of an electron on a single site, and a term proportional to $t$ which is the kinetic energy gain which comes from sharing the electron between two sites. The factor of $\cos (\theta / 2)$ comes from the $S U(2)$ rotation. Using the relation $\cos \theta=\vec{S}_{1} \cdot \vec{S}_{2} / S^{2}$, and a standard trigonometric identity for double angles we can write the energy of this state as

$$
E_{0} \approx-\frac{t}{4 S^{2}} \vec{S}_{1} \cdot \vec{S}_{2}+\text { const }
$$

The kinetic energy of the itinerant electron generates an effective FM Heisenberg exchange interaction $J_{D E}=t / 4 S^{2}$ between the two sites. The generalization of this semiclassical analysis to the lattice [3], gives an effective Hamiltonian for the DEFM

$$
\mathcal{H}_{\text {eff }}=\mathcal{H}_{\rho}+\mathcal{H}_{\sigma}
$$

with independent spin and charge excitations

$$
\mathcal{H}_{\rho}=-t \sum_{\langle i j\rangle} f_{i}^{\dagger} f_{j} \quad \mathcal{H}_{\sigma}=-J_{D E} \sum_{\langle i j\rangle} \vec{S}_{i} \cdot \vec{S}_{j}
$$

Here the sum $\sum_{\langle i j\rangle}$ runs over nearest neighbour sites, and $f_{i}^{\dagger}$ is the creation operator for an electron aligned with the local moment on site $i$. The charge part of the Hamiltonian describes electrons with a tight binding band dispersion $\varepsilon_{k}=-z t \gamma_{k}$ and the spin Hamiltonian has spinwave excitations with dispersion $\omega_{q}=z J_{D E} S\left[1-\gamma_{q}\right]$ where $\gamma_{k}=\left(\cos k_{x}+\cos k_{y}+\cos k_{z}\right) / 3$ is the structure factor for the cubic lattice of Mn sites in units where the lattice constant $a=1$. The size of the effective exchange interaction $J_{D E}$ is determined by the ground state average of the itinerant electron electron kinetic energy in the FM ground state of the model, and so is also proportional to $t$

$$
J_{D E}=\frac{t}{4 S^{2}} \sum_{\langle i j\rangle}\left\langle f_{i}^{\dagger} f_{j}\right\rangle_{0}
$$

In fact the energy scales of both spin and charge excitations must be be proportional to $t$, since the role of hopping is to lift the degeneracy between the many different charge configurations for a given doping and spin state.

Using this effective Hamiltonian, we can understand both the metallic conduction of the FM phase of the CMR Manganites, and the fact that their spinwave spectrum, when measured in inelastic neutron scattering experiments, looks a lot like that of a FM nearest neighbour Heisenberg model. However we have neglected all quantum fluctuation 
effects, which means we cannot explain, for example, the large damping of spinwaves which is also observed in Neutron scattering experiments. The most important feature missing from this Hamiltonian is the interaction between spin and charge excitations, which is present when fluctuation effects are taken into account. It is this interaction vertex which we now calculate. However, in order to do this, we must first re-derive the semi-classical treatment of the DEFM in a rigorously controlled manner. Since the literature on the DE model is too large to review in this format, we will not make any attempt to discuss the alternative approaches which might be taken to this problem.

\section{NEW SPINWAVE EXPANSION}

Since the Hund's rule coupling $J$ is much larger than the kinetic energy scale $t$, we begin by setting $t=0$ and considering the atomic limit of the Kondo lattice Hamiltonian Eqn. 11. It is our aim to first diagonalize the local Kondo interaction, before reintroducing the kinetic energy terms. Physically, it makes no sense to distinguish between the magnetic excitations of the local moment $\vec{S}$ and the spin of the itinerant electron $\vec{s}$, so we consider instead the total spin $\vec{T}=\vec{S}+\vec{S}$. In terms of this coordinate, the Hamiltonian Eqn. 1 for a single sight can be written

$$
-J \vec{S} . \vec{s}=-\frac{J}{2}\left[\vec{T}^{2}-\vec{S}^{2}-\vec{s}^{2}\right]=-\frac{J}{2}[T(T+1)-S(S+1)-s(s+1)]
$$

Here $S$ is the size of the local moment, $s$ is the spin quantum number for the itinerant electron, and the total spin quantum number $T$ can take on values $T=S \pm s$. There are four possible states. The lowest energy state has the eigenvalue $E=-J S / 2$. It corresponds to one itinerant electron being perfectly aligned with the local moment to give a total spin $T=S+1 / 2$. There are two states with eigenvalue $E=0$ and total spin $T=S$. In these the itinerant electron orbital is either empty or doubly occupied. There is also a high energy state with eigenvalue $E=J(S+1) / 2$, and total spin eigenvalue $T=S-1 / 2$. This has one itinerant electron perfectly "anti-aligned" with the local moment.

Now we introduce electron operators which create these states, when acting on a vacuum in which the local moment has some definite $z$-component $S^{z}$. We introduce one Fermi operator $\left\{f, f^{\dagger}\right\}=1$ to describe high spin states, and another Fermi operator $\left\{p, p^{\dagger}\right\}=1$ to describe low spin states. These are illustrated in cartoon form in Fig. 6 . Next we need to introduce a bosonic description of the spin algebra of the total spin $\vec{T}$. We do this by generalizing the usual Holstein-Primakoff transformation used to describe rotations to spins of a fixed length [ $₫$ ] to the case in which the length of the spin is an operator.

$$
\begin{aligned}
T^{z}=S^{z}+\frac{u^{\dagger} u-d^{\dagger} d}{2} & =T-\tilde{a}^{\dagger} \tilde{a} \\
T^{+}=S^{+}+u^{\dagger} d & =\sqrt{2 T} \sqrt{1-\frac{\tilde{a}^{\dagger} \tilde{a}}{2 T} \tilde{a}}
\end{aligned}
$$




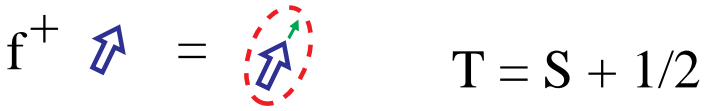

$$
\begin{aligned}
& \mathrm{p}^{+} \text {公遥, } \mathrm{T}=\mathrm{S}-1 / 2
\end{aligned}
$$

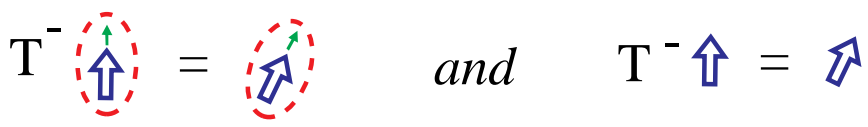

$$
\begin{aligned}
& \mathrm{T}^{+} \text {迹, 㗄 etc. }
\end{aligned}
$$

FIGURE 6. Cartoon illustration of new operator coordinates.

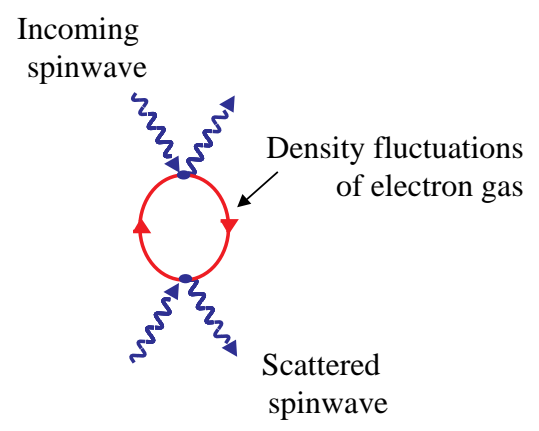

FIGURE 7. Interaction between spinwaves mediated by density fluctuations of the electron gas.

where

$$
T=S+\frac{f^{\dagger} f-p^{\dagger} p}{2}
$$

The physical meaning of these spin operator is illustrated in Fig. 6. Finally, we expand the operators $\{f, p, \tilde{a}\}$ in terms of the operators $\{u, d, a\}$, where $\left[a, a^{\dagger}\right]=1$ is the Holstein-Primakoff Boson for the core spin $\vec{S}$. We do this order by order in the small parameter $1 / \sqrt{S}$. We must make sure that all the correct canonical (anti-)commutation relations are conserved by this transformation at each order. At the end of all this hard work, we are left with an inverse transformation

$$
c_{\uparrow}=f\left(1-\frac{\tilde{a}^{\dagger} \tilde{a}+p^{\dagger} p}{4 S}\right)-\frac{p \tilde{a}^{\dagger}}{\sqrt{2 S}}+O\left(1 / S^{3 / 2}\right)
$$




$$
c_{\downarrow}=p\left(1-\frac{\tilde{a}^{\dagger} \tilde{a}+f f^{\dagger}}{4 S}\right)+\frac{f \tilde{a}}{\sqrt{2 S}}+O\left(1 / S^{3 / 2}\right)
$$

which can be substituted into the kinetic energy term in Eqn. 1 to give the interaction between spin and charge excitations. In the limit $J \rightarrow \infty$ the high energy " $p$ " states can be neglected entirely. In terms of the new coordinates, the effective Hamiltonian Eqn. 4 reads

$$
\mathcal{H}_{\rho}=\sum_{k} \varepsilon_{k} f_{k}^{\dagger} f_{k} \quad \mathcal{H}_{\sigma}=\sum_{q} \omega_{q} \tilde{a}_{q}^{\dagger} \tilde{a}_{q}
$$

The spin and charge modes now interact according to

$$
\begin{aligned}
& \mathcal{V}=\frac{1}{N} \sum_{k_{1} \ldots k_{4}} v_{24}^{13}: f_{1}^{\dagger} f_{2}: \tilde{a}_{3}^{\dagger} \tilde{a}_{4} \delta_{1+3-2-4}+\ldots \\
& v_{24}^{13}=\frac{1}{4\left(S+\frac{1}{2}\right)}\left[\left(1+\frac{1}{8 S}\right)\left(\varepsilon_{1+3}+\varepsilon_{2+4}\right)-\left(\varepsilon_{1}+\varepsilon_{2}\right)\right]
\end{aligned}
$$

Here the charge density : $f^{\dagger} f$ : is measured relative to the average density of itinerant electrons, and corrections to the vertex at $O\left(1 / S^{2}\right)$ have been suppressed. The form of the vertex is physically correct - spin and charge quantum numbers are independently conserved (as they must be in the limit $J \rightarrow \infty$ ), and the strength of their interaction is proportional to $t$. The consequence of this vertex is an effective interaction between charge and spin modes at $O\left(1 / S^{2}\right)$, shown in Fig 77. These results were first introduced in [5]. Further details of the transformation, including terms up $O\left(1 / S^{2}\right)$, are given in [6] and [7].

\section{RESULTS FOR THE DOUBLE EXCHANGE FERROMAGNET}

We now briefly illustrate some of the consequences of the interaction between spin and charge modes for the physical properties of the DEFM. All examples given are for a system with a density of $x=0.3$ holes per site, spin $S=3 / 2$ and hopping integral $t=1$. These values are chosen to correspond with the FM state of $\mathrm{La}_{1-x} \mathrm{Ca}_{x} \mathrm{MnO}_{3}$ with maximumal $T_{c}$. Since the Hamiltonian is quadratic in Fermi and Bose fields, and the commutation relatations of all operators are well defined, standard diagramatic techniques can be used to calculate the spin and charge response functions of the model.

In Fig. 8 the semi-classical spinwave spectrum $\omega_{q}$ of the DEFM is shown, together with the renormalized spectrum when the leading quantum corrections to the spinwave dispersion at are taken into account. The effect of the interaction is large, especially at the zone boundary. The spinwave over all scale of spinwave dispersion is reduced, and no longer has the simple form associated with a nearest neighbour Heisenberg FM. Physically, this is because quantum fluctuations about the FM groundstate generate effective non nearest neighbour exchange couplings.

Spinwaves excitations are exact eigenstates of the Heisenberg FM, but are damped in the DEFM. This damping arises because spinwaves can decay into lower energy spin 


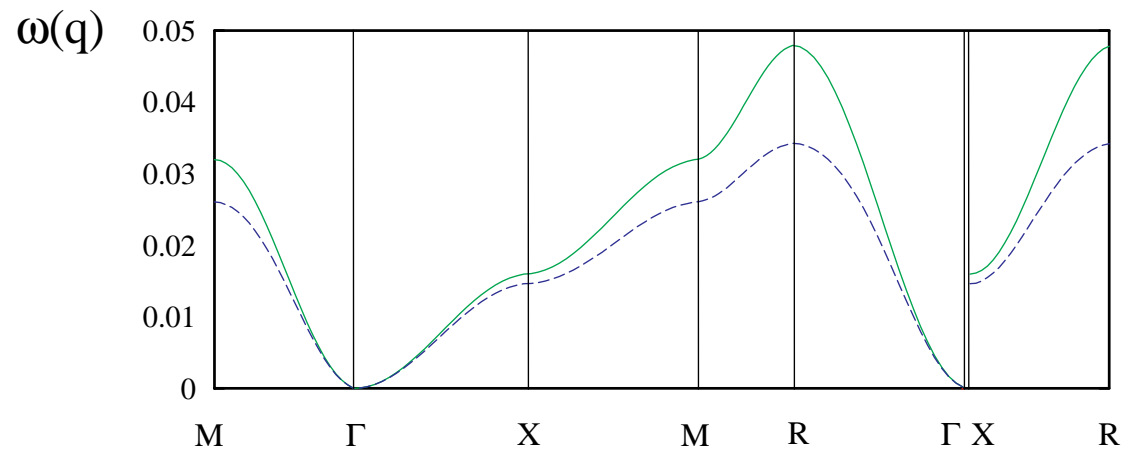

FIGURE 8. Spinwave spectrum of the DEFM at zero temperature. Upper solid line - semi-classical result throughout the Brillouin zone. Lower dashed line - result when leading quantum corrections are taken into account.

excitations, dressed with charge density fluctuations (electron-hole pairs). This damping is also large, as is illustrated in Fig. 9

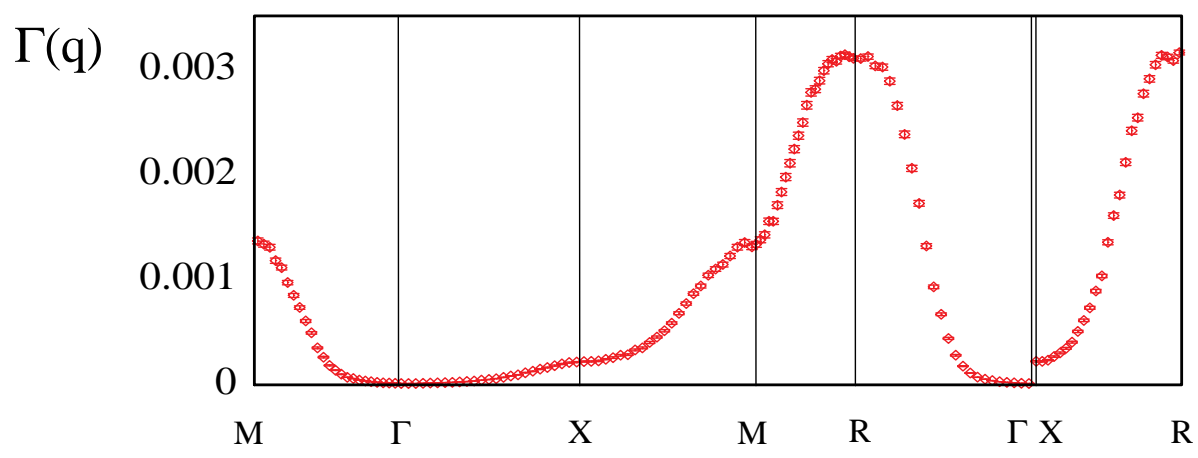

FIGURE 9. Damping of spinwaves in the DEFM throughout the Brillouin zone.

Temperature corrections to the spinwave dispersion in a Heisenberg FM are exactly proportional to the zero temperature dispersion, and scale as $T^{5 / 2}$ at low temperatures. Temperature corrections in the DEFM are substantially enhanced, compared with the semi-classically equivalent Heisenberg FM, and are nolonger simply proportional to the zero temperature dispersion. The comparison of the two models is illustrated in Fig. 10 . Detailed results will be presented in a forthcoming publication [7].

\section{CONCLUSIONS}

We have developed a controlled large $S$ expansion of the Kondo Lattice model, based on local eigenstates of total spin. This approach can applied to any magnetically ordered state, but is particularly convenient when working in the "double exchange" limit $J \gg$ 


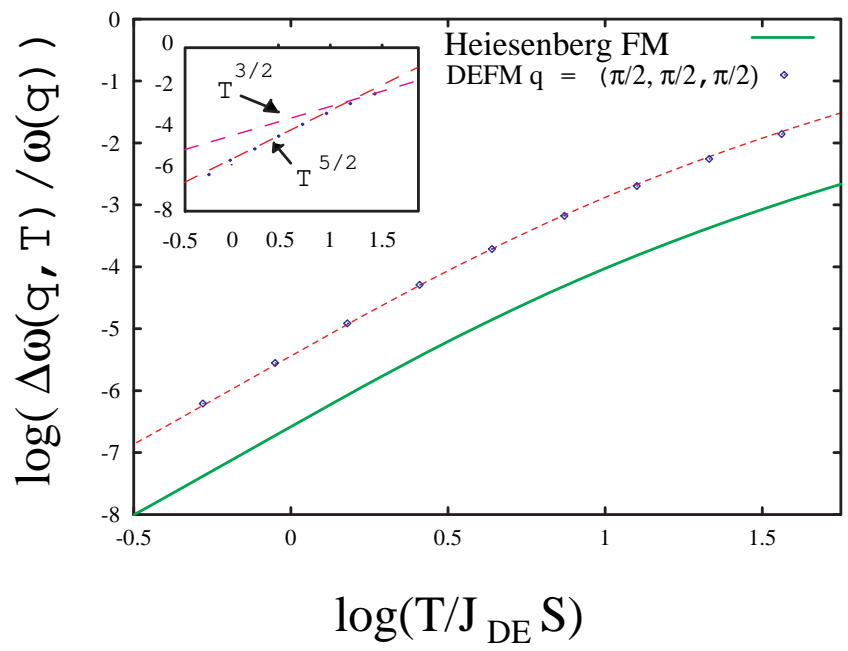

FIGURE 10. Log-log plot of finite temperature corrections to the spinwave spectrum of the DEFM, together with those of the semi-classically equivalent Heisenberg FM. Temperature corrections $\Delta \omega(q, T)$ are normalized to bare dispersion $\omega(q)$, temperatures to the scale of spinwave dispersion $J_{D E} S$.

$t$. This approach has been applied to the double exchange ferromagnet, which has been shown to behave in a quite different way from the Heisenberg ferromagnet when quantum effects are correctly included.

\section{ACKNOWLEDGMENTS}

It is our pleasure to acknowledge the collaboration of Andrey V. Chubukov in this work, and support under NSF grant DMR-9632527 and the visitor's program of MPI-PKS Dresden. We would like also to express our gratitude to Adolfo Avella and the organizers of the Vietri training course.

\section{REFERENCES}

1. Zener, C., Phys. Rev., 82, 403 (1951).

2. Anderson, P. W., and Hasegawa, H., Phys. Rev., 100, 675 (1955).

3. de Gennes, P. G., Phys. Rev., 118, 141-154 (1960).

4. Holstein, and Primakoff, Phys. Rev., 58, 1098-1113 (1940).

5. Shannon, N., and Chubukov, A., cond-mat/0011390 - J. Phys.: Condens. Matter, 14, L235-241 (2002).

6. Shannon, N., J. Phys.: Condens. Matter, 13, 6371-6386 (2001).

7. Shannon, N., and Chubukov, A., Phys. Rev. B, 65, 104418 (2002). 